\title{
Cálculo de la reactividad utilizando cuadraturas de Newton-Cotes de siete puntos
}

\author{
Daniel Suescún-Díaz, Jesús A. Chala-Casanova y Luis E. Cardoso-Páez \\ Departamento de Ciencias Naturales, Universidad Surcolombiana, Neiva, Huila, Colombia. \\ (correo-e: daniel.suescun@usco.edu.co, chalarock@gmail.com, luispaez0203@gmail.com)
}

Recibido May. 6, 2021; Aceptado Jul. 1, 2021; Versión final Jul. 26, 2021, Publicado Oct. 2021

\section{Resumen}

En este estudio se resuelve numéricamente la ecuación inversa de la cinética puntual, utilizando la cuadratura de Newton-Cotes de siete puntos para calcular la reactividad. El método se valida realizando diferentes experimentos numéricos, considerando diferentes formas de la densidad de la población de neutrones con diferentes tiempos de simulación. Los resultados muestran que los experimentos numéricos permiten calcular la reactividad con alta precisión con respecto a la solución de referencia dada por la solución analítica. Se concluye que los resultados son muy precisos, si los pasos de tiempo son preferiblemente pequeños, cuando se comparan con otros métodos reportados en la literatura.

Palabras clave: reactores nucleares; reactividad nuclear; densidad de neutrones; cuadratura Newton-Cotes; simulación numérica

\section{Reactivity calculation using the seven-point Newton-Cotes quadrature}

\begin{abstract}
In this study, the inverse point kinetics equation is solved numerically by using the seven-point Newton-Cotes quadrature to calculate reactivity. Different numerical experiments considering different shapes of neutron population density with different simulation times are performed to validate the methodology. The results show that the numerical experiments performed allow calculating reactivity with high accuracy for the reference solution given by the analytical solution. It is concluded that the results obtained are highly accurate, if time steps are preferably small, when compared to other methods reported in the literature.
\end{abstract}

Keywords: nuclear reactors; nuclear reactivity; neutron density; Newton-Cotes quadrature; numerical simulation 


\section{INTRODUCCIÓN}

Los métodos numéricos son frecuentemente usados para realizar simulaciones en diferentes aplicaciones como en el comportamiento térmico (Ortega et al., 2009), la propagación de una fisura en el material (Palma et al., 2010), en la solución de ecuaciones de advección-difusión no estacionarias (Bermúdez y Juárez,2014), y el efecto de la presión en el proceso de mezcla metano-oxígeno (De la Cruz et al., 2015). En física de reactores nucleares se usan para resolver las ecuaciones de la cinética puntual, es un conjunto de ecuaciones diferenciales en general no lineales, fuertemente acopladas que presentan el fenómeno de rigidez (Chao y Attard, 1985). Estas ecuaciones permiten cuantificar la cantidad de neutrones producidos por eventos de fisión. La reacción de fisión es una de la más importantes en física nuclear que se puede controlar por medio de un reactor nuclear. Las ecuaciones de la cinética puntual son un modelo útil para estudiar el comportamiento temporal de la densidad de la población de neutrones y la densidad de la concentración de precursores (Duderstadt y Hamilton, 1976). Además, las ecuaciones de la cinética puntual pueden ser invertidas para calcular la reactividad. La reactividad es quizás el parámetro más importante en un reactor nuclear, es por esto por lo que el cálculo preciso de la reactividad puede ser la principal preocupación de los físicos e ingenieros de reactores.

Muchos métodos han sido desarrollados para calcular la reactividad con el objetivo de ser implementado en medidores digitales, algunos trabajos consideran: La ecuación directa de la cinética puntual, despejando el parámetro de la reactividad usando diferencias finitas, considerando un filtro pasa bajo (Shimazu et al., 1987). Hay trabajos que realizan simulaciones considerando la ecuación inversa de la cinética puntual con fuente de neutrones (Hoogenboom y Van der Sluijs, 1988). Otro método, calcula la reactividad discretizando la ecuación inversa de la cinética puntual considerando solo dos puntos para la densidad de la población de neutrones (Binney y Bakir, 1989). Unos meses después, un método diseña un medidor digital de reactividad usando una interpolación lineal (Ansari, 1991). Otros trabajos se han enfocado en el desarrollo de métodos numéricos que aumentan la precisión para el cálculo de la reactividad, una utiliza la ecuación inversa con tres y cinco puntos mediante los polinomios de Lagrange (Malmir y Vosoughi, 2013), el método directo de las ecuaciones de la cinética puntual denominado formulación matricial (Suescún-Díaz et al., 2018), el método de la transformada wavelet (Rasak y Rathinasamy, 2018), el uso de transporte hibrido ( Picca y Furfaro, 2018), la reconstrucción de la reactividad por medio de detectores de neutrones (Chentre et al., 2019), un poco más reciente, se utiliza el método con infinitos números de Bernoulli (Suescún-Díaz et al., 2020). En este trabajo se propone un nuevo método para el cálculo de la reactividad usando una cuadratura de Newton-Cotes considerando un polinomio interpolador de siete puntos usando la ecuación inversa, sin considerar la fuente de neutrones ni ruidos en la densidad de la población de neutrones.

\section{OTROS ANTECEDENTES}

Las ecuaciones de la cinética puntual están dadas por el siguiente conjunto de ecuaciones (Stacey, 2018):

$$
\begin{aligned}
& \frac{d}{d t} n(t)=\frac{\rho(t)-\beta}{\Lambda} n(t)+\sum_{i=1}^{m} \lambda_{i} C_{i} \\
& \frac{d}{d t} C_{i}(t)=\frac{\beta_{i}}{\Lambda} n(t)-\lambda_{i} C_{i}
\end{aligned}
$$

Dónde $n(t)$ es la densidad de la población de neutrones, $\rho(t)$ es la reactividad, $C_{i}(t)$ es la concentración de precursores del i-ésimo grupo de neutrones atrasados, $\Lambda$ es el tiempo de generación de neutrones instantáneos, $\beta$ es la fracción total de neutrones atrasados, $m$ el número del grupo de neutrones precursores, $\beta_{i}$ es la i-ésima fracción de neutrones atrasados y $\lambda_{i}$ es la constante de decaimiento del i-ésimo grupo de precursores. La ecuación inversa de la cinética puntual se obtiene al resolver (2) que son un conjunto de $m$ ecuaciones diferenciales asociadas a las concentraciones de los distintos grupos de precursores, luego esta solución se sustituye en la ecuación (1) y finalmente se resuelve para $\rho(t)$,

$$
\rho(t)=\beta+\frac{\Lambda}{n} \frac{d}{d t} n(t)-\frac{n(0)}{n(t)} \sum_{i=1}^{m} \beta_{i} e^{-\lambda_{i} t}-\frac{1}{n(t)} \sum_{i=1}^{m} \beta_{i} \lambda_{i} \int_{0}^{t} e^{-\lambda_{i}\left(t-t^{\prime}\right)} n\left(t^{\prime}\right) d t^{\prime}
$$

La integral contenida en la ecuación (3) es una integral de la forma de convolución y se debe resolver considerando todos los valores de la densidad de la población de neutrones para cada instante de tiempo $t$, 
lo que genera un alto costo computacional para tiempos de medición grandes. En este trabajo, se propone resolver numéricamente la integral dependiente de la densidad de la población de neutrones contenida en la ecuación (3). La solución numérica se da por medio de reglas compuestas a partir de cuadraturas de NewtonCotes de siete puntos. Estas cuadraturas dan como resultado un esquema que permite calcular la reactividad en instantes de tiempo discretos, además entre mayor sea el grado de la cuadratura mayor será el orden de precisión. La ecuación (3) puede ser evaluada aproximadamente haciendo $\mathrm{t}=\mathrm{t}_{\mathrm{k}}, \mathrm{t}^{\prime}=\mathrm{t}_{\mathrm{z}}$ y el paso del cálculo $\Delta \mathrm{t} \approx$ dt, obteniendo:

$$
\rho\left(t_{k}\right) \approx \beta+\frac{\Lambda}{n\left(t_{k}\right)}\left[\frac{n\left(t_{k}\right)-n\left(t_{k-1}\right)}{\Delta t}\right]-\frac{n\left(t_{0}\right)}{n\left(t_{k}\right)} \sum_{i=1}^{m} \beta_{i} e^{-\lambda_{i} t_{k}}-\frac{1}{n\left(t_{k}\right)} \sum_{i=1}^{m} \beta_{i} \lambda_{i} \int_{0}^{t_{k}} e^{-\lambda_{i}\left(t_{k}-t_{z}\right)} n\left(t_{z}\right) d t_{z}
$$

Sea considerado el integrando en la ecuación (4) como:

$$
\int_{0}^{t_{k}} e^{-\lambda_{i}\left(t_{k}-t_{z}\right)} n\left(t_{z}\right) d t_{z}=\int_{0}^{t_{k}} f\left(t_{z}\right) d t_{z}
$$

Sí se usa un polinomio de Lagrange $P_{N}\left(t_{z}\right)$, dónde $N$ indica el grado del polinomio para interpolar la función contenida en el integrando; entonces, es posible reescribir el lado derecho de la ecuación (5) de la siguiente forma (Stoer y Bulirsch, 2010),

$$
\int_{0}^{t_{N}} f\left(t_{z}\right) d t_{z} \approx \int_{0}^{N} P_{N}\left(t_{z}\right) d t_{z}=\int_{0}^{N} \sum_{p=0}^{N} f\left(t_{p}\right) \prod_{\substack{q=0 \\ q \neq p}}^{N} \frac{t_{z}-t_{q}}{t_{p}-t_{q}} d t_{z}
$$

Asumiendo, físicamente que los periodos de medición de la densidad de la población de neutrones son constantes o que las abscisas se encuentran igualmente espaciadas y asumiendo además $d t_{k}=d t_{z} \approx d t$, entonces se puede escribir,

$$
\begin{aligned}
& t_{z}=t_{0}+z \Delta t \\
& t_{q}=t_{0}+q \Delta t \\
& t_{p}=t_{0}+p \Delta t
\end{aligned}
$$

Para $\mathrm{N}=6$, se reescribe la ecuación (6) como,

$$
\int_{0}^{t_{6}} P_{6}\left(t_{z}\right) d t_{z}=\sum_{p=0}^{6} f\left(t_{p}\right) \int_{0}^{6} \prod_{\substack{q=0 \\ q \neq p}}^{6} \frac{z-q}{p-q} d t_{z}
$$

La ecuación (8) representa el área bajo la curva de un polinomio interpolador de sexto grado que pasa a través de 7 puntos. Expandiendo la sumatoria, se obtiene:

$$
\sum_{p=0}^{6} f\left(t_{p}\right) \int_{0}^{6} \prod_{\substack{q=0 \\
q \neq p}}^{6} \frac{z-q}{p-q} d t_{z} \approx \Delta t_{z}\left[\begin{array}{c}
\frac{41}{140} f\left(t_{0}\right)+\frac{54}{35} f\left(t_{1}\right)+\frac{27}{140} f\left(t_{2}\right)+\frac{68}{35} f\left(t_{3}\right) \\
+\frac{27}{140} f\left(t_{4}\right)+\frac{54}{35} f\left(t_{5}\right)+\frac{41}{140} f\left(t_{6}\right)
\end{array}\right]
$$

Donde el incremento $\Delta t_{z}$ es la versión discreta del diferencial $d t_{z}$, los coeficientes que acompañan a cada una de la función evaluada en los diferentes puntos del polinomio, los términos a la derecha en la ecuación (9) puede ser escrita en una forma más compacta como: 


$$
\int_{0}^{t_{6}} P_{6}(t) d t \approx \sum_{s=0}^{6} C_{s} f\left(t_{s}\right) \Delta t_{z}
$$

Donde los coeficientes $\mathrm{C}_{\mathrm{s}}$ son dados por,

$$
C_{0}=C_{6}=\frac{41}{140}, \quad C_{1}=C_{5}=\frac{54}{35}, \quad C_{2}=C_{4}=\frac{27}{140}, \quad C_{3}=\frac{68}{35}
$$

Es posible elaborar una regla compuesta para adaptar la cuadratura para intervalos más generales de la siguiente forma,

$$
\begin{aligned}
\int_{0}^{t_{k}} f\left(t_{z}\right) d t_{z}=\sum_{z=1}^{\frac{k}{6}} \int_{0}^{6} P_{6}\left(t_{z}\right) d t_{z} & =\int_{0}^{t_{6}} P_{6}\left(t_{z}\right) d t_{z}+\ldots+\int_{t_{i}}^{t_{i+6}} P_{6}\left(t_{z}\right) d t_{z}+\ldots+\int_{t_{k-6}}^{t_{k}} P_{6}\left(t_{z}\right) d t_{z} \\
& \approx \sum_{z=1}^{\frac{k}{6}} \sum_{s=0}^{6} C_{s} f\left(t_{6 z-s}\right) \Delta t_{z}
\end{aligned}
$$

Usando la ecuación (11), el integrando en la ecuación (4) se puede reescribir de la siguiente forma,

$$
\int_{0}^{t_{k}} e^{-\lambda_{i}\left(t_{k}-t_{z}\right)} n\left(t_{z}\right) d t_{z}=\int_{0}^{t_{k}} f\left(t_{z}\right) d t_{z} \approx \sum_{z=1}^{\frac{k}{6}} \sum_{s=0}^{6} C_{s} f\left(t_{6 z-s}\right) \Delta t_{z}=\sum_{z=1}^{\frac{k}{6}} \sum_{s=0}^{6} C_{s} e^{-\lambda_{i}\left(t_{k}-t_{6 z-s}\right)} n\left(t_{6 z-s}\right) \Delta t
$$

Sustituyendo la ecuación (12) en la ecuación inversa de la cinética puntual dada por la ecuación (4), la reactividad se puede escribir finalmente,

$$
\begin{aligned}
\rho\left(t_{k}\right) & \approx \beta+\frac{\Lambda}{n\left(t_{k}\right)}\left[\frac{n\left(t_{k}\right)-n\left(t_{k-1}\right)}{\Delta t}\right]-\frac{n\left(t_{0}\right)}{n\left(t_{k}\right)} \sum_{i=1}^{m} \beta_{i} e^{-\lambda_{i} t_{k}} \\
& -\frac{\Delta t}{n\left(t_{k}\right)} \sum_{i=1}^{m} \sum_{z=1}^{\frac{k}{6}} \sum_{s=0}^{6} \beta_{i} \lambda_{i} C_{s} e^{-\lambda_{i}\left(t_{k}-t_{6 z-s}\right)} n\left(t_{6 z-s}\right)
\end{aligned}
$$

La ecuación (13) representa el método propuesto para calcular la reactividad nuclear mediante la cuadratura de Newton-Cotes con siete puntos. Para el valor de la reactividad se requieren 7 muestras de la densidad de la población de neutrones, produciendo un atraso. Sin embargo, esto no sería ninguna dificultad ya que en la práctica se tiene un gran número de valores de la densidad de la población de neutrones.

\section{RESULTADOS}

Para realizar las simulaciones numéricas se consideran los parámetros físicos de un reactor térmico con Uranio-235, los valores de los parámetros utilizados son: $\beta_{i}=\{0.000266,0.001491,0.001316,0.002849$, $0.000896,0.000182\}, \beta=\Sigma \beta_{i}=0.007, \lambda_{i}=\{0.0127,0.0317,0.115,0.311,1.4,3.87\} \mathrm{s}^{-1}$ y $\Lambda=5 \times 10^{-5} \mathrm{~s}$. Asumiendo que la forma de la densidad de la población de neutrones es conocida sin presentar fluctuaciones, $n(t)$ puede ser representada por medio de una función, es posible resolver la integral de convolución contenida en la ecuación integro diferencial dada en (3) de forma analítica. En la Tabla 1 se muestran siete funciones elementales que permiten simular la forma de la densidad de la población de neutrones y se presenta el resultado analítico calculando la integral de convolución en cada caso. Para cada forma existe un indicador $f_{j}$ para identificarla. Para $j=1$ se hace referencia al experimento numérico considerando que la densidad de la población de neutrones se asume exponencial $e^{\omega t}$. Dicha solución es usada finalmente para el cálculo de la reactividad y obtener el método de referencia para comparar con el método propuesto. 
Tabla 1. Formas para la densidad de población de neutrones y las soluciones analíticas para la integral de la ecuación inversa de la cinética puntual.

\begin{tabular}{|l|l|r|}
\hline$f_{j}$ & $\mathrm{n}\left(\mathrm{t}_{k}\right)$ & $\int_{0}^{t_{k}} e^{-\lambda_{i}\left(t_{k}-t_{z}\right)} n\left(t_{z}\right) d t_{z}$ \\
\hline 1 & $e^{\omega t_{k}}$ & $\frac{e^{\omega t_{k}}-e^{-\lambda_{i} t_{k}}}{\lambda_{i}+\omega}$ \\
\hline 2 & $a+\omega t_{k}$ & $\frac{a}{\lambda_{i}}\left(1-e^{-\lambda_{i} \mathrm{t}}\right)+\frac{\omega}{\lambda_{i}^{2}}\left[\lambda_{i} t-1+e^{\left.-\lambda_{i} \mathrm{t}\right]}\right.$ \\
\hline 3 & $a+\omega t_{k}^{2}$ & $\frac{a}{\lambda_{i}}\left(1-e^{-\lambda_{i} \mathrm{t}}\right)+\frac{\omega}{\lambda_{i}^{3}}\left[\lambda_{i} t\left(\lambda_{i} t-2\right)+2-2 e^{-\lambda_{i} \mathrm{t}}\right]$ \\
\hline 4 & $a+b \sin \left(\omega t_{k}\right)$ & $\frac{a}{\lambda_{i}}\left(1-e^{-\lambda_{i} \mathrm{t}}\right)+\frac{b}{\lambda_{i}^{2}+\omega^{2}}\left[\lambda_{i} \sin (\omega t)-\omega \cos (\omega t)+\omega e^{\left.-\lambda_{i} \mathrm{t}\right]}\right.$ \\
\hline 5 & $a+b \cos \left(\omega t_{k}\right)$ & $\frac{a}{\lambda_{i}}\left(1-e^{-\lambda_{i} \mathrm{t}}\right)+\frac{b}{\lambda_{i}^{2}+\omega^{2}}\left[\omega \sin (\omega t)+\lambda_{i} \cos (\omega t)-\lambda_{i} e^{\left.-\lambda_{i} \mathrm{t}\right]}\right.$ \\
\hline 6 & $a+b \sinh \left(\omega t_{k}\right)$ & $\frac{a}{\lambda_{i}}\left(1-e^{-\lambda_{i} \mathrm{t}}\right)+\frac{b}{\lambda_{i}^{2}-\omega^{2}}\left[\lambda_{i} \sinh (\omega t)-\omega \cosh (\omega t)+\omega e^{\left.-\lambda_{i} \mathrm{t}\right]}\right.$ \\
\hline 7 & $a+b \cosh \left(\omega t_{k}\right)$ & $\frac{a}{\lambda_{i}}\left(1-e^{-\lambda_{i} \mathrm{t}}\right)+\frac{b}{\lambda_{i}^{2}-\omega^{2}}\left[\lambda_{i} \cosh (\omega t)-\omega \sinh (\omega t)-\lambda_{i} e^{\left.-\lambda_{i} \mathrm{t}\right]}\right.$ \\
\hline
\end{tabular}

Estas funciones elementales asumidas requieren parámetros adicionales $a$ y $b$, las cuales pueden tomar diferentes valores según el experimento numérico. Los valores de $\omega$, se define como un vector que almacena los distintos valores que corresponden a las raíces positivas de la "ecuación en horas" (Kinard y Allen, 2004): $\omega=\left[\omega_{1}, \omega_{2}, \omega_{3}, \omega_{4}, \omega_{5}, \omega_{6}\right]=[0.00243,0.01046,0.02817,0.12353,1.00847,2.345]$. Para validar el método propuesto, la primera serie de experimentos numéricos resuelve la ecuación inversa de la cinética puntual usando como entrada las siete distintas formas de la densidad de la población de neutrones, tres distintos pasos de tiempo $\Delta \mathrm{t}=0.5 \mathrm{~s}, \Delta \mathrm{t}=0.1 \mathrm{~s}$ y $\Delta \mathrm{t}=0.01 \mathrm{~s}$, con $a=1, b=\pi / 10$ y seis valores de $\omega$. Las Tablas $2-3$ presentan las máximas diferencias en función del parámetro $\omega_{m}$ que producen reactividades hasta $300 \mathrm{pcm}$ (partes por cien mil) en caso de que la variación de la densidad de neutrones sea de tipo exponencial. Los resultados indican que a medida que se disminuye el paso del cálculo, las máximas diferencias decaen significativamente, pero manteniendo aproximadamente el mismo orden de precisión, siendo este una característica muy marcada en la dependencia del método propuesto.

Para pasos de tiempo pequeños las máximas diferencias son también muy pequeñas. Sin embargo, hay una dificultad la cual resulta ser el costo computacional ya que se requieren evaluar tres sumas en la ecuación (13). La primera y tercera suma no es problema pues dependen máximo de seis puntos dados por el número de precursores, la sumatoria que depende del tiempo ( $t_{k}$ ) es una convolución, siendo este costo muy alto que puede limitar el uso cuando aumente el número de muestras, debido que es necesario usar todos los valores o el histórico de la densidad de la población de neutrones en cada instante de tiempo.

Tabla 2. Máximas diferencias (en $p c m$ ) para las distintas formas de la densidad de neutrones, distintos pasos de tiempo, $t=300$ s, usando $\omega_{1}$ y $\omega_{2}$

\begin{tabular}{|c|l|c|c|c|c|c|}
\hline \multirow{2}{*}{$f_{j}$} & \multicolumn{5}{|c|}{$\omega_{1}$} & \multicolumn{3}{c|}{$\omega_{2}$} \\
\cline { 2 - 7 } & $\Delta \mathrm{t}=0.5$ & $\Delta \mathrm{t}=0.1$ & $\Delta \mathrm{t}=0.01$ & $\Delta \mathrm{t}=0.5$ & $\Delta \mathrm{t}=0.1$ & $\Delta \mathrm{t}=0.01$ \\
\hline 1 & $3.19 \times 10^{-1}$ & $8.24 \times 10^{-6}$ & $8.59 \times 10^{-12}$ & $3.22 \times 10^{-1}$ & $8.36 \times 10^{-6}$ & $8.68 \times 10^{-12}$ \\
\hline 2 & $3.19 \times 10^{-1}$ & $8.24 \times 10^{-6}$ & $8.67 \times 10^{-12}$ & $3.22 \times 10^{-1}$ & $8.35 \times 10^{-6}$ & $8.85 \times 10^{-12}$ \\
\hline 3 & $4.98 \times 10^{-1}$ & $1.99 \times 10^{-5}$ & $1.09 \times 10^{-11}$ & $4.98 \times 10^{-1}$ & $1.99 \times 10^{-5}$ & $1.09 \times 10^{-11}$ \\
\hline 4 & $3.19 \times 10^{-1}$ & $8.22 \times 10^{-6}$ & $8.67 \times 10^{-12}$ & $3.20 \times 10^{-1}$ & $8.26 \times 10^{-6}$ & $8.94 \times 10^{-12}$ \\
\hline 5 & $3.19 \times 10^{-1}$ & $8.21 \times 10^{-6}$ & $8.67 \times 10^{-12}$ & $3.18 \times 10^{-1}$ & $8.21 \times 10^{-6}$ & $8.67 \times 10^{-12}$ \\
\hline 6 & $3.19 \times 10^{-1}$ & $8.22 \times 10^{-6}$ & $8.67 \times 10^{-12}$ & $3.22 \times 10^{-1}$ & $8.33 \times 10^{-6}$ & $8.85 \times 10^{-12}$ \\
\hline 7 & $3.19 \times 10^{-1}$ & $8.22 \times 10^{-6}$ & $8.67 \times 10^{-12}$ & $3.22 \times 10^{-1}$ & $8.33 \times 10^{-6}$ & $8.75 \times 10^{-12}$ \\
\hline
\end{tabular}


Tabla 3. Máximas diferencias (en $p c m$ ) para las distintas formas de la densidad de neutrones, distintos pasos de tiempo, $t=300 \mathrm{~s}$, usando $\omega_{3}$ y $\omega_{4}$

\begin{tabular}{|c|c|c|l|c|l|c|}
\hline \multirow{2}{*}{$f_{j}$} & \multicolumn{3}{|c|}{$\omega_{3}$} & \multicolumn{3}{c|}{$\omega_{4}$} \\
\cline { 2 - 7 } & $\Delta \mathrm{t}=0.5$ & $\Delta \mathrm{t}=0.1$ & $\Delta \mathrm{t}=0.01$ & $\Delta \mathrm{t}=0.5$ & $\Delta \mathrm{t}=0.1$ & $\Delta \mathrm{t}=0.01$ \\
\hline 1 & $3.28 \times 10^{-1}$ & $8.62 \times 10^{-6}$ & $8.87 \times 10^{-12}$ & $3.61 \times 10^{-1}$ & $1.01 \times 10^{-5}$ & $9.32 \times 10^{-12}$ \\
\hline 2 & $3.27 \times 10^{-1}$ & $8.58 \times 10^{-6}$ & $8.59 \times 10^{-12}$ & $3.48 \times 10^{-1}$ & $9.65 \times 10^{-6}$ & $8.68 \times 10^{-12}$ \\
\hline 3 & $4.98 \times 10^{-1}$ & $1.99 \times 10^{-5}$ & $1.09 \times 10^{-11}$ & $4.98 \times 10^{-1}$ & $1.99 \times 10^{-5}$ & $1.09 \times 10^{-11}$ \\
\hline 4 & $3.22 \times 10^{-1}$ & $8.34 \times 10^{-6}$ & $8.50 \times 10^{-11}$ & $3.32 \times 10^{-1}$ & $8.81 \times 10^{-6}$ & $8.76 \times 10^{-12}$ \\
\hline 5 & $3.22 \times 10^{-1}$ & $8.34 \times 10^{-6}$ & $8.67 \times 10^{-12}$ & $3.32 \times 10^{-1}$ & $8.81 \times 10^{-6}$ & $9.02 \times 10^{-12}$ \\
\hline 6 & $3.28 \times 10^{-1}$ & $8.62 \times 10^{-6}$ & $8.78 \times 10^{-12}$ & $3.61 \times 10^{-1}$ & $1.01 \times 10^{-5}$ & $9.44 \times 10^{-12}$ \\
\hline 7 & $3.28 \times 10^{-1}$ & $8.62 \times 10^{-6}$ & $8.92 \times 10^{-12}$ & $3.61 \times 10^{-1}$ & $1.01 \times 10^{-5}$ & $9.44 \times 10^{-12}$ \\
\hline
\end{tabular}

La Figura 1 se presenta el cálculo de la reactividad en pcm para una forma de la densidad de la población de neutrones $n(t)=a+b t^{2}$ con un paso dt=0.5 s, se alcanza un pico de $428 \mathrm{pcm}$, en un tiempo de 4.7 segundos, decreciendo a menos de $350 \mathrm{pcm}$ en un tiempo de $15 \mathrm{~s}$, en todo el tiempo de simulación se presenta una máxima diferencia de 0.49 pcm.

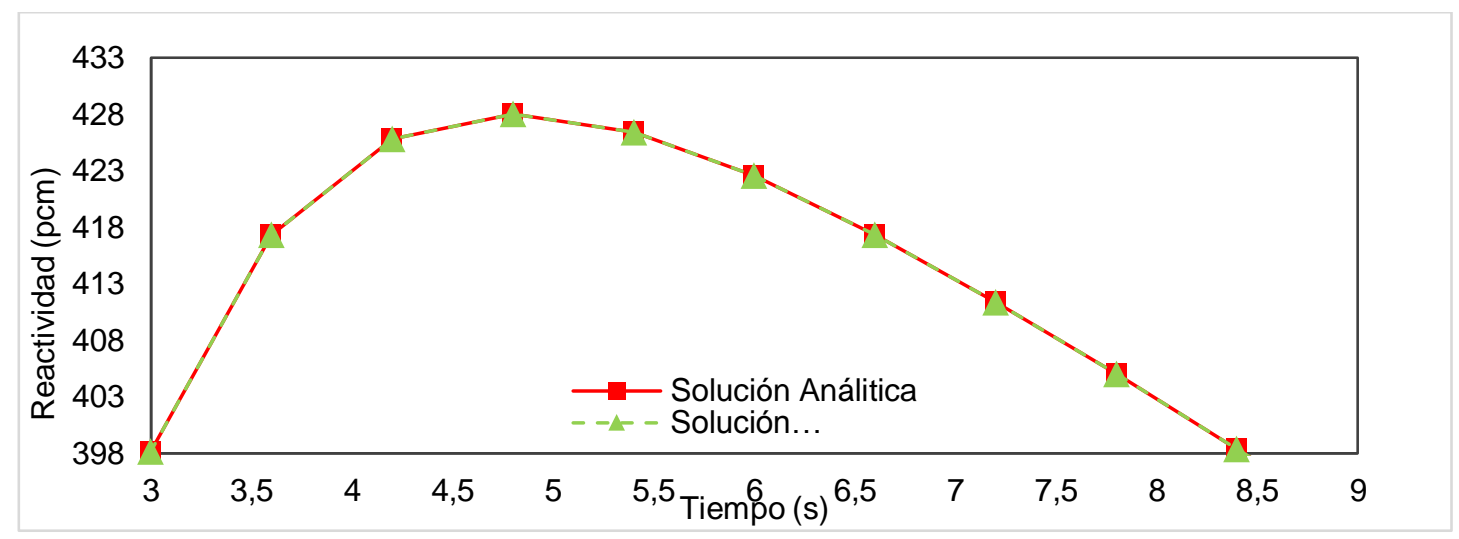

Fig. 1. Comparación del cálculo de la reactividad entre la solución analítica y numérica para $n(t)=a+b t^{2}$

Considerando valores $\omega>1$, la forma exponencial de la densidad de la población de neutrones, se puede alcanzar valores de reactividad de $550 \mathrm{pcm}$ aproximadamente, las máximas diferencias varían casi un orden de magnitud con respecto al experimento numérico anterior como se presenta en la Tabla 4. Sin embargo, los resultados obtenidos con el método propuesto mantienen una buena precisión, aunque el valor de $\omega$ aumente considerablemente.

Tabla 4. Máximas diferencias (en $p c m$ ) para las distintas formas de la densidad de neutrones, distintos pasos de tiempo, $t=300 s$, con $\omega_{5}$ y $\omega_{6}$

\begin{tabular}{|c|c|c|c|c|c|c|}
\hline \multirow{2}{*}{$f_{j}$} & \multicolumn{3}{|c|}{$\omega_{5}$} & \multicolumn{3}{c|}{$\omega_{6}$} \\
\cline { 2 - 7 } & $\Delta \mathrm{t}=0.5$ & $\Delta \mathrm{t}=0.1$ & $\Delta \mathrm{t}=0.01$ & $\Delta \mathrm{t}=0.5$ & $\Delta \mathrm{t}=0.1$ & $\Delta \mathrm{t}=0.01$ \\
\hline 1 & $7.82 \times 10^{-1}$ & $3.82 \times 10^{-5}$ & $1.30 \times 10^{-11}$ & $2.01 \times 10^{0}$ & $1.88 \times 10^{-4}$ & $1.57 \times 10^{-11}$ \\
\hline 2 & $4.00 \times 10^{-1}$ & $1.57 \times 10^{-5}$ & $8.59 \times 10^{-12}$ & $4.14 \times 10^{-1}$ & $2.03 \times 10^{-5}$ & $8.68 \times 10^{-12}$ \\
\hline 3 & $4.98 \times 10^{-1}$ & $1.99 \times 10^{-5}$ & $1.09 \times 10^{-11}$ & $4.98 \times 10^{-1}$ & $1.99 \times 10^{-5}$ & $1.09 \times 10^{-11}$ \\
\hline 4 & $4.40 \times 10^{-1}$ & $1.40 \times 10^{-5}$ & $1.00 \times 10^{-11}$ & $7.63 \times 10^{-1}$ & $2.30 \times 10^{-5}$ & $1.04 \times 10^{-11}$ \\
\hline 5 & $4.40 \times 10^{-1}$ & $1.40 \times 10^{-5}$ & $9.69 \times 10^{-12}$ & $7.66 \times 10^{-1}$ & $2.30 \times 10^{-5}$ & $1.06 \times 10^{-11}$ \\
\hline 6 & $7.82 \times 10^{-1}$ & $3.82 \times 10^{-5}$ & $1.30 \times 10^{-11}$ & $2.01 \times 10^{0}$ & $1.88 \times 10^{-4}$ & $1.57 \times 10^{-11}$ \\
\hline 7 & $7.82 \times 10^{-1}$ & $3.82 \times 10^{-5}$ & $1.30 \times 10^{-11}$ & $2.01 \times 10^{0}$ & $1.88 \times 10^{-4}$ & $1.57 \times 10^{-11}$ \\
\hline
\end{tabular}

En la Tabla 5 se comparan las máximas diferencias obtenidas con: un polinomio de Lagrange con cinco puntos (Malmir y Vosoughi, 2013), Formulación matricial (Suescún-Díaz et al., 2018) y el método propuesto. En estos experimentos se utilizan distintos parámetros y dos formas de la densidad de la población de neutrones. Los resultados muestran que las máximas diferencias obtenidas con el método propuesto de Newton-Cotes de 7 puntos el más preciso de los tres métodos en el cálculo de la reactividad para este tipo de forma de la densidad de la población de neutrones. 
Tabla 5. Máximas diferencias obtenidas en experimentos numéricos con $\Delta t=0.1$

\begin{tabular}{|c|c|c|c|c|c|c|}
\hline $\mathrm{n}(\mathrm{t})$ & $\mathrm{a}$ & $\mathrm{B}$ & $\mathrm{t}(\mathrm{s})$ & Lagrange 5-Puntos & $\mathrm{FM}$ & Newton-Cotes 7 Puntos \\
\hline $\mathrm{a}+\mathrm{bt}_{\mathrm{k}}$ & 1 & 1 & 10 & $6.15 \times 10^{-2}$ & $5.12 \times 10^{-2}$ & $1.57 \times 10^{-5}$ \\
\hline $\mathrm{a}+\operatorname{sen}\left(\mathrm{bt}_{\mathrm{k}}\right)$ & 1 & $\pi / 10$ & 180 & $2.79 \times 10^{-2}$ & $4.22 \times 10^{-2}$ & $6.55 \times 10^{-4}$ \\
\hline
\end{tabular}

En la Tabla 6 se compara el método propuesto con el método de la formulación matricial; se observa que para pasos de tiempo grandes el método sigue dando buenos resultados, además, cuando el paso de tiempo es del orden de las centésimas, el método propuesto logra máximas diferencias con valores de menor orden de magnitud que la formulación matricial.

Tabla 6. Máximas diferencias para $\mathrm{j}=1, \omega_{5}, t=80 \mathrm{~s}$

\begin{tabular}{|c|c|c|}
\hline$\Delta \mathrm{t}$ & $\mathrm{FM}$ & Newton-Cotes 7 Puntos \\
\hline 0.01 & $3.34 \times 10^{-11}$ & $3.41 \times 10^{-12}$ \\
\hline 0.05 & $4.77 \times 10^{-12}$ & $1.83 \times 10^{-7}$ \\
\hline 0.1 & $4.66 \times 10^{-12}$ & $3.82 \times 10^{-5}$ \\
\hline
\end{tabular}

La Tabla 7 presenta las máximas diferencias en la reactividad para las diferentes formas de la densidad de la población de neutrones entre el método propuesto y la solución analítica dada por la ecuación (3). Los valores medios $\mu_{\omega}$ y la desviación estándar $\sigma_{\omega}$ de estas diferencias dependen del parámetro $\omega_{m}$. Para pasos $\Delta \mathrm{t}=0.5 \mathrm{~s}$, $\Delta \mathrm{t}=0.1 \mathrm{~s}$ y $\Delta \mathrm{t}=0.01 \mathrm{~s}$, se encuentran unas máximas diferencias en promedio en el cálculo de la reactividad, independiente de la forma de la densidad de la población de neutrones de $5.35 \times 10^{-1}, 2.54 \times 10^{-5}$ y $1.00 \times 10^{-11}$ pcm, con una desviación estándar promedio de $3.45 \times 10^{-1}, 3.38 \times 10^{-5}$ y $1.48 \times 10^{-12} \mathrm{pcm}$ respectivamente.

Tabla 7. Valores medios y desviaciones estándar de las máximas diferencias

\begin{tabular}{|c|c|c|c|c|c|c|}
\hline & \multicolumn{2}{|c|}{$\Delta \mathrm{t}=0.5$} & \multicolumn{2}{c|}{$\Delta \mathrm{t}=0.1$} & \multicolumn{2}{c|}{$\Delta \mathrm{t}=0.01$} \\
\cline { 2 - 7 } & $\mu_{\omega}$ & $\sigma_{\omega}$ & $\mu_{\omega}$ & $\sigma_{\omega}$ & $\mu_{\omega}$ & $\sigma_{\omega}$ \\
\hline$\omega_{1}$ & $3.44 \times 10^{-1}$ & $6.76 \times 10^{-2}$ & $9.89 \times 10^{-6}$ & $4.41 \times 10^{-6}$ & $8.98 \times 10^{-12}$ & $8.50 \times 10^{-13}$ \\
\hline$\omega_{2}$ & $3.46 \times 10^{-1}$ & $6.69 \times 10^{-2}$ & $9.96 \times 10^{-6}$ & $4.38 \times 10^{-6}$ & $9.09 \times 10^{-12}$ & $8.06 \times 10^{-13}$ \\
\hline$\omega_{3}$ & $3.50 \times 10^{-1}$ & $6.51 \times 10^{-2}$ & $1.02 \times 10^{-5}$ & $4.30 \times 10^{-6}$ & $9.03 \times 10^{-12}$ & $8.40 \times 10^{-13}$ \\
\hline$\omega_{4}$ & $3.70 \times 10^{-1}$ & $5.77 \times 10^{-2}$ & $3.94 \times 10^{-6}$ & $3.94 \times 10^{-6}$ & $9.36 \times 10^{-12}$ & $7.47 \times 10^{-13}$ \\
\hline$\omega_{5}$ & $5.89 \times 10^{-1}$ & $1.83 \times 10^{-1}$ & $2.55 \times 10^{-5}$ & $1.21 \times 10^{-5}$ & $1.12 \times 10^{-11}$ & $1.81 \times 10^{-12}$ \\
\hline$\omega_{6}$ & $1.21 \times 10^{0}$ & $7.61 \times 10^{-1}$ & $9.28 \times 10^{-5}$ & $8.90 \times 10^{-5}$ & $1.25 \times 10^{-11}$ & $3.05 \times 10^{-12}$ \\
\hline
\end{tabular}

En el último experimento numérico la dependencia de la densidad de la población de neutrones es dada por $n(t)=a-b \operatorname{sinc}(\omega t)$. En la Figura 2 se presenta la reactividad con el método propuesto, presentando un pico de $120 \mathrm{pcm}$ en un tiempo $\mathrm{t}=10 \mathrm{~s}$, cuyo valor empieza a disminuir lentamente hasta aproximadamente un valor cercano a cero en un tiempo $t=100 \mathrm{~s}$. Confiando en los resultados obtenidos en los anteriores experimentos numéricos, suponiendo que el método funcione en forma adecuada, las diferencias en la reactividad tienen una máxima diferencia de $1.0 \times 10^{-11} \mathrm{pcm}$, valor muy pequeño y nos muestra como el método propuesto puede ser considerado como un método alternativo para el cálculo de la reactividad en reactores nucleares.

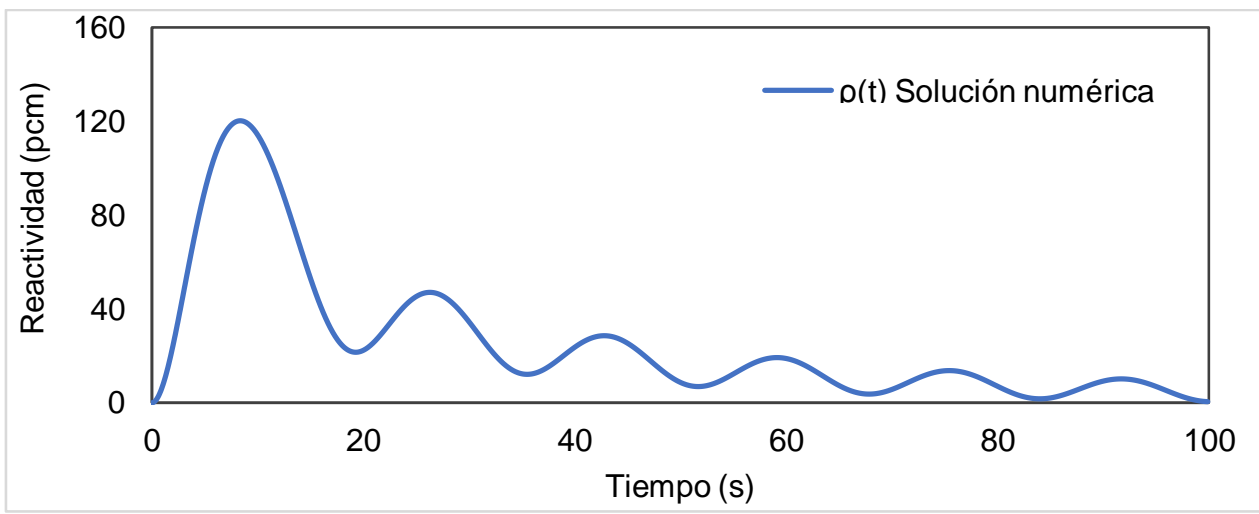

Fig. 2. Reactividad para la forma de la densidad de neutrones $n(t)=a-b \operatorname{Sin} c(\omega t)$ con $a=1, b=\pi / 10$ y $d t=0.01 s$ 


\section{CONCLUSIONES}

Se presentó un método para el cálculo de la reactividad utilizando una cuadratura de Newton-Cotes de siete puntos mediante un polinomio de Lagrange de sexto grado que permiten resolver numéricamente la ecuación inversa la de la cinética puntual. El método es de fácil implementación y permite calcular la reactividad con una alta presión sin importar la forma de la densidad de la población de neutrones, el método obtiene máximas diferencias dependiendo directamente del valor del paso del cálculo seleccionado, siendo posible determinar con anticipación el error máximo cometido. Los diferentes experimentos numéricos permiten considerar el método propuesto como un método alternativo para ser implementado en medidores de reactividad digital.

\section{REFERENCIAS}

Ansari, S.A., Development of on-line Reactivity Meter for Nuclear Reactors, https://doi.org/10.1109/23.83857, IEEE Trans. Nuc.I Sci., 38(4), 946-952 (1991)

Bermúdez, B., y Juárez, L., Solución Numérica de una Ecuación del Tipo Advección-Difusión, http://dx.doi.org/10.4067/S0718-07642014000100016, Información Tecnológica., 25(1), 151-160 (2014)

Binney, S.E., y Bakir A.J., Design and Development of a Personal-Computer-Based Reactivity Meter for a Research Reactor, https://doi.org/10.13182/nt89-a34223, Nucl. Technol., 85(1), 12-21 (1989)

Chao, Y.A., y Attard A., A Resolution of the Stiffness Problem of Reactor Kinetics, https://doi.org/10.13182/nse85-7, Nucl. Sci. Eng., 90(1), 40-46 (1985)

Chentre, N., Saracco, P., Dulla., S y Ravetto, P., On the Prompt Time Eigenvalue Estimation for Subcritical Multiplying Systems, https://doi.org/10.1016/j.anucene.2019.04.030, Ann. Nucl. Energy., 132, 172-180 (2019)

De la Cruz, M., Polupan, G., Martínez, E., y Carvajal, I., Estudio Numérico del Efecto de la Presión en el Proceso de Mezcla Metano-Oxígeno en un Arreglo de Chorros 4-Lug Bolt, http://dx.doi.org/10.4067/S0718-07642015000200018, Información Tecnológica., 26(2), 153-162 (2015)

Duderstadt, J.J., y Hamilton L.J., Nuclear Reactor Analysis, John Wiley and Sons, Nueva York, Estados Unidos (1976)

Hetrick, D. L., Dynamics of Nuclear Reactors, American Nuclear Society, Illinois, Estados Unidos (1993)

Hoogenboom, J. E., y Van Der Sluijs A.R., Neutron Source Strength Determination for on-line Reactivity Measurements, https://doi.org/10.1016/0306-4549(88)90059-x, Ann. Nucl. Energy., 15(12), 553-559 (1988)

Kinard, M., y Allen E.J., Efficient Numerical Solution of the Point Kinetics Equations in Nuclear Reactor Dynamics, https://doi.org/10.1016/j.anucene.2003.12.008, Ann. Nucl. Energy., 31(9), 1039-1051 (2004)

Malmir, H., y Vosoughi N., On-line Reactivity Calculation Using Lagrange Method,

https://doi.org/10.1016/j.anucene.2013.07.006, Ann. Nucl. Energy., 62, 463-467 (2013)

Marguet, S., The Physics of Nuclear Reactors, Springer, Cham, Suiza (2017)

Mohideen, A. R. M., y Rathinasamy, N., Haar Wavelet for Solving the Inverse Point Kinetics Equations and Estimation of Feedback Reactivity Coefficient under Background Noise, https://doi.org/10.1016/j.anucene.2018.02.033, https://doi.org/10.1016/j.nucengdes.2018.04.022, Nuclear Engineering and Design., 335, 202-209 (2018)

Ortega, A.J., Pires, L.F.G., y Nieckele, A.O., Una Alternativa para la Simulación Numérica del Comportamiento Térmico en Régimen Transitorio de Flujo de Gas en Redes de Ductos, http://dx.doi.org/10.4067/S0718-07642009000500011, Información Tecnológica., 20(5), 83-88 (2009)

Palma, J.A., Barbosa, J.M.A., y Silva, A.A., Simulación Numérica de la Propagación de una Fisura en un Material Degradado por Efecto de la Fragilización por Hidrógeno, http://dx.doi.org/10.4067/S0718-07642010000200009, Información Tecnológica., 21(2), 65-76 (2010)

Picca, P., y Furfaro, R., Reactivity determination using the hybrid transport point kinetics and the area method. https://doi.org/10.1016/j.anucene.2017.12.019, Ann. Nucl. Energy, 114, 191-197 (2018)

Shimazu, Y., Nakano Y., Tahara Y., y Okayama T., Development of a Compact Digital Reactivity Meter and a Reactor Physics Data Processor, https://doi.org/10.13182/nt87-a33964, Nucl. Technol., 77(3), 247-254 (1987)

Stacey, W.M., Nuclear Reactor Physics, Wiley-VCH, Weinheim, Alemania (2018)

Stoer, J., y Bulirsch R., Introduction to Numerical Analysis, Springer, Nueva York (2011)

Suescún-Díaz, D., Cabrera-Capera E., y Lozano-Parada J. H., Matrix Formulation for the Calculation of Nuclear Reactivity, https://doi.org/10.1016/j.anucene.2018.02.033, Ann. Nucl. Energy., 116, 137-142 (2018)

Suescún-Díaz, D., Ule-Duque, G., y Escobar, F.H., Novel approach to solving the inverse equation of point kinetics by the Bernoulli number generalisation method, https://doi.org/10.1080/00223131.2020.1742813,

J. Nucl. Scien. Technol., 57(8), 989-999 (2020) 